\title{
Advances in high-resolution ultrasound-modulated optical tomography in biological tissues
}

Sava Sakadzic, Lihong V. Wang

Sava Sakadzic, Lihong V. Wang, "Advances in high-resolution ultrasoundmodulated optical tomography in biological tissues," Proc. SPIE 5697, Photons Plus Ultrasound: Imaging and Sensing 2005: The Sixth Conference on Biomedical Thermoacoustics, Optoacoustics, and Acousto-optics, (25 April 2005); doi: 10.1117/12.589411

SPIE. Event: SPIE BiOS, 2005, San Jose, CA, United States 


\title{
Advances in high-resolution ultrasound-modulated optical tomography in biological tissues
}

\author{
Sava Sakadžić and Lihong V. Wang \\ Optical Imaging Laboratory, Department of Biomedical Engineering, Texas A\&M University, \\ College Station, Texas 77843-3120
}

\begin{abstract}
We present an extension of our work on implementation of high-resolution ultrasound-modulated optical tomography that, based on optical contrast, can image several millimeters deep into soft biological tissues. A long-cavity confocal Fabry-Perot interferometer, which provides a large etendue and a short response time, was used to detect the ultrasound-modulated coherent light that traversed the scattering biological tissue. Using $15-\mathrm{MHz}$ ultrasound, light absorbing structures placed $>3 \mathrm{~mm}$ below the surface of chicken breast tissue were imaged with high contrast. The resolutions along the axial and lateral directions with respect to the ultrasound propagation direction were better than $70 \mu \mathrm{m}$ and $120 \mu \mathrm{m}$, respectively. The resolutions can be scaled down further by using higher ultrasound frequencies. This technology is complementary to other imaging technologies, such as confocal microscopy and optical-coherence tomography, and has potential for broad biomedical applications.
\end{abstract}

Keywords: ultrasonic modulation, acousto-optical tomography, multiply scattered light, microscopy

\section{INTRODUCTION}

Great effort has been made in the recent past to develop new imaging modalities based on the optical properties of soft biological tissues in the visible and near-infrared regions. At these wavelengths, radiation is nonionizing and the optical properties of biological tissues are related to the molecular structure, offering potential for the detection of functions and abnormalities.

Ultrasound-modulated optical tomography ${ }^{1,2}$ is a hybrid technique, proposed to provide better resolution for the optical imaging of soft biological tissue by combining ultrasonic resolution and optical contrast. Collective motions of the optical scatterers and periodic changes in the optical index of refraction are generated by ultrasound to produce fluctuations in the intensity of the speckles that are formed by the multiple-scattered light. $^{3-5}$ The ultrasound-modulated component of light carries information about the optical properties of tissue from the region of interaction between the optical and ultrasonic waves. However, it is a challenge to detect this modulated component efficiently because of diffused light propagation and uncorrelated phases among individual speckles. Several schemes of detection ${ }^{2,3,6-14}$ have been explored. A CCD camera that provides parallel speckle detection $^{8,9,14}$ was used to produce a better SNR than a single square-law detector. To obtain resolution along the ultrasonic axis, several groups explored various techniques, including ultrasound frequency sweep ${ }^{7}$ computer tomography, ${ }^{15}$ and tracking of ultrasound pulses ${ }^{11}$ or short bursts. ${ }^{13}$ The pulsed ultrasound approaches provide direct resolution along the ultrasonic axis and are more compatible with conventional ultrasound imaging. Pulsed ultrasound can have a much higher instantaneous power than $\mathrm{CW}$ ultrasound, reducing the undesired effect of the increased noise due to its wide bandwidth.

In this Letter, for the first time to our knowledge, we report high-resolution ultrasound-modulated optical imaging using a long-cavity confocal Fabry-Perot interferometer (CFPI). ${ }^{16}$ Our CFPI has a greater etendue - defined as the product of the acceptance solid angle and the area - than most CCD cameras and provides parallel speckle processing. In addition, CFPI can detect the propagation of high-frequency ultrasound pulses in real time and tolerate speckle decorrelation. CFPI is especially efficient at high ultrasound frequencies, where the background light can be filtered out effectively while the ultrasound-modulated component is transmitted. Using our setup, optical features of $\sim 100 \mu \mathrm{m}$ in size embedded more than $3 \mathrm{~mm}$ below the surface of chicken breast tissue were resolved with high contrast in both the axial and lateral directions. 

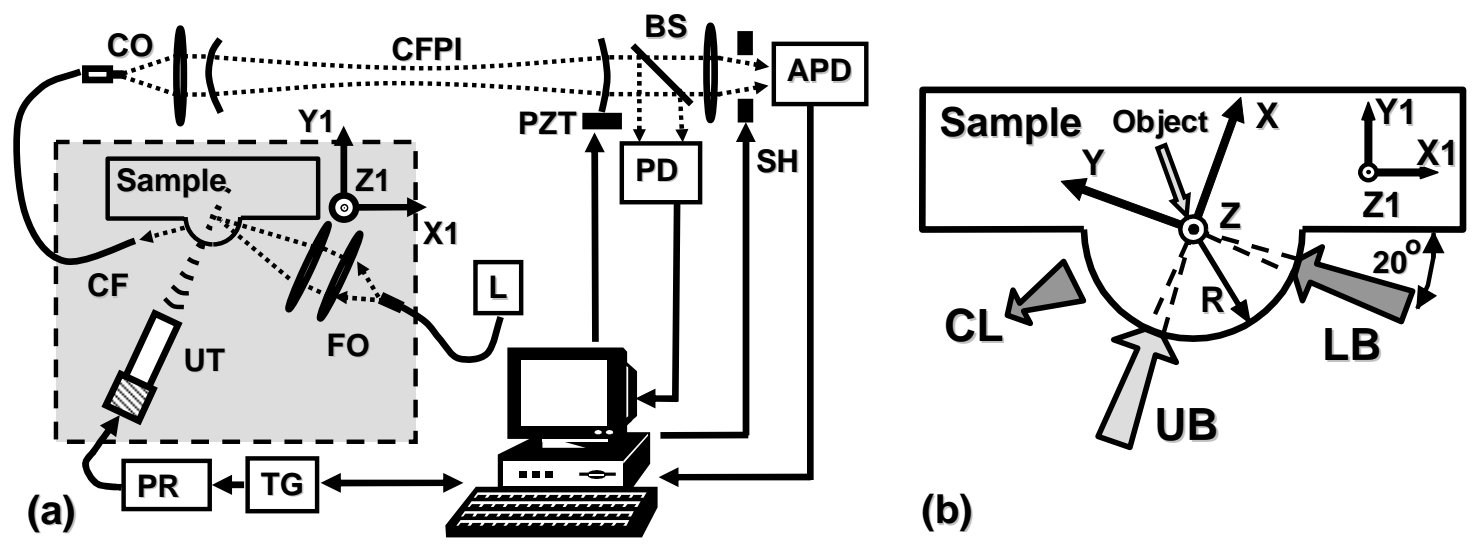

(b)

Figure 1. (a) Schematic of the experimental setup: L, laser; TG, trigger generator; PR, pulser/receiver; UT, ultrasonic transducer; FO, focusing optics; CF, collecting fiber; CO, coupling optics; CFPI, confocal Fabry-Perot interferometer; BS, beam splitter; APD, avalanche photo-diode; SH, shutter; PD, photo detector. (b) Top view of the sample: UB, ultrasound beam; LB, incident light beam; CL, collected light; R, radius.

\section{EXPERIMENTAL SETUP}

The experimental setup is shown in Fig. 1. Samples were gently pressed through a slit along the $\mathrm{Z}$ axis to create a semi-cylindrical bump. The orthogonal ultrasonic and optical beams [Fig. 1(b)] were focused to the same spot below the sample surface. Diffusely transmitted light was collected by an optical fiber with a $600-\mu \mathrm{m}$ core diameter. This configuration minimized the contribution of unmodulated light from the shallow regions to the background and in addition enhanced the interaction between the ultrasound and some quasi-ballistic light that still existed at small imaging depths (up to one transport mean free path).

A focused ultrasound transducer (Ultran, $15-\mathrm{MHz}$ central frequency, 4.7-mm lens diameter, 4.7-mm focal length, 15-MHz estimated bandwidth) was driven by a pulser (GE Panametrics, 5072PR). The ultrasound focal peak pressure was 3.9 $\mathrm{MPa}$, within the ultrasound safety limit at this frequency for tissues without well-defined gas bodies. ${ }^{17}$ The laser light (Coherent, Verdi; 532-nm wavelength) was focused onto a spot of $\sim 100 \mu \mathrm{m}$ in diameter below the surface of an otherwise scattering-free sample. The optical power delivered to the sample was $100 \mathrm{~mW}$. Although the CW power in this proof-of-principle experiment exceeded the safety limit for average power, the duration of the light exposure to the sample can be reduced to only a few $\mu$ s for each ultrasound pulse propagation through the region of interest, and, therefore, the safety limit will not be exceeded in practice even if the focus is maintained in a scattering medium. The sample was mounted on a three-axis (X1, Y1, and Z1) translational stage. The ultrasound transducer and the sample were immersed in water for acoustic coupling. The light focusing optics and the collecting fiber were also immersed in the same water tank. The collected light was coupled into the CFPI that was operated in transmission mode $\left(50-\mathrm{cm}\right.$ cavity length, $0.1-\mathrm{mm}^{2} \mathrm{sr}$ etendue, and $>20$ finesse). The light sampled by the beam splitter was used in a cavity tuning procedure. The cavity was first swept through one free spectral range to find the position of the central frequency of the unmodulated light. Then, one CFPI mirror was displaced by a calibrated amount such that the cavity was tuned to the frequency of one sideband of the ultrasound-modulated light (15 MHz greater than the laser light frequency). An avalanche photo-diode (APD) (Advanced Photonix) acquired the light filtered by the interferometer, and the signal was sampled at $100 \mathrm{Ms} / \mathrm{sec}$ with a data acquisition board (GAGE, CS14100). A computer program written in LabView controlled the movement of the CFPI mirror and the other sequences of the control signals.

A trigger generator (Stanford Research, DG535) triggered both the ultrasound pulse generation and the data acquisition from the APD. Since the resonant frequency of the CFPI cavity coincided with one sideband of the ultrasound-modulated light, the signal acquired by the APD during the ultrasound propagation through the sample represented the distribution of the ultrasound-modulated optical intensity along the ultrasonic axis and, therefore, yielded a one-dimensional (1D) image. In each operational cycle, the resonant frequency of the CFPI was first tuned, and then data from 4000 ultrasound pulses were acquired in one second. Averaging over 10 


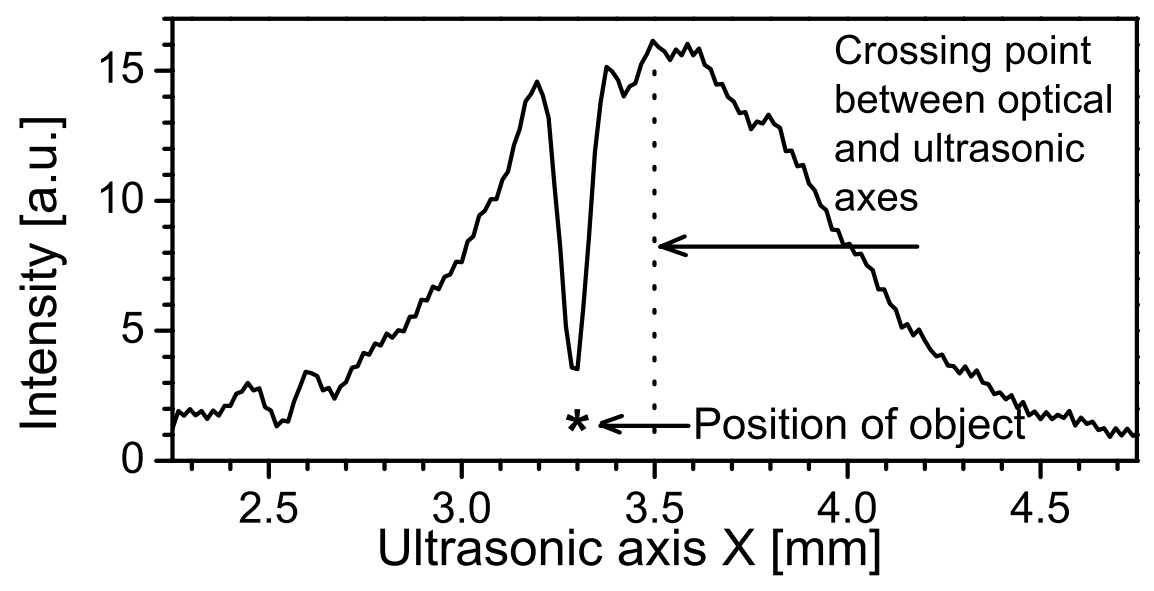

Figure 2. Temporal dependence of the ultrasound-modulated light intensity during the propagation of an ultrasound pulse through the sample.

cycles was usually necessary to obtain a satisfactory SNR for each 1D image. Two-dimensional images were obtained by scanning the sample along the $\mathrm{Z}$ direction and acquiring each corresponding $1 \mathrm{D}$ image.

\section{RESULTS}

Figure 2 presents a typical profile of the temporal dependence of the ultrasound-modulated light intensity during an ultrasound pulse propagation through the sample. The time of propagation was multiplied by $1500 \mathrm{~ms}^{-1}$, the approximate speed of sound in the sample, to be converted into distance along the $\mathrm{X}$ axis, where the origin corresponded to the trigger for the signal acquisition from the APD. The sample, made of chicken breast tissue, was pressed through the 4-mm wide slit. A long rod of $60 \mu \mathrm{m}$ in diameter, made from black latex, which was transparent for ultrasound but absorptive for light, was placed below the sample surface along the $\mathrm{Z}$ axis of the cylindrical tissue bump of a 2 -mm radius. Because the profiles of the optical radiance and the ultrasound intensity within the sample determined the distribution of the ultrasound-modulated optical intensity, the maximum corresponded to the crossing point between the optical and the ultrasonic axes as indicated in Fig. 2. The differences between the optical properties of the object and the tissue created a deep dip in the ultrasound-modulated light intensity when the ultrasound pulse passed through the object.
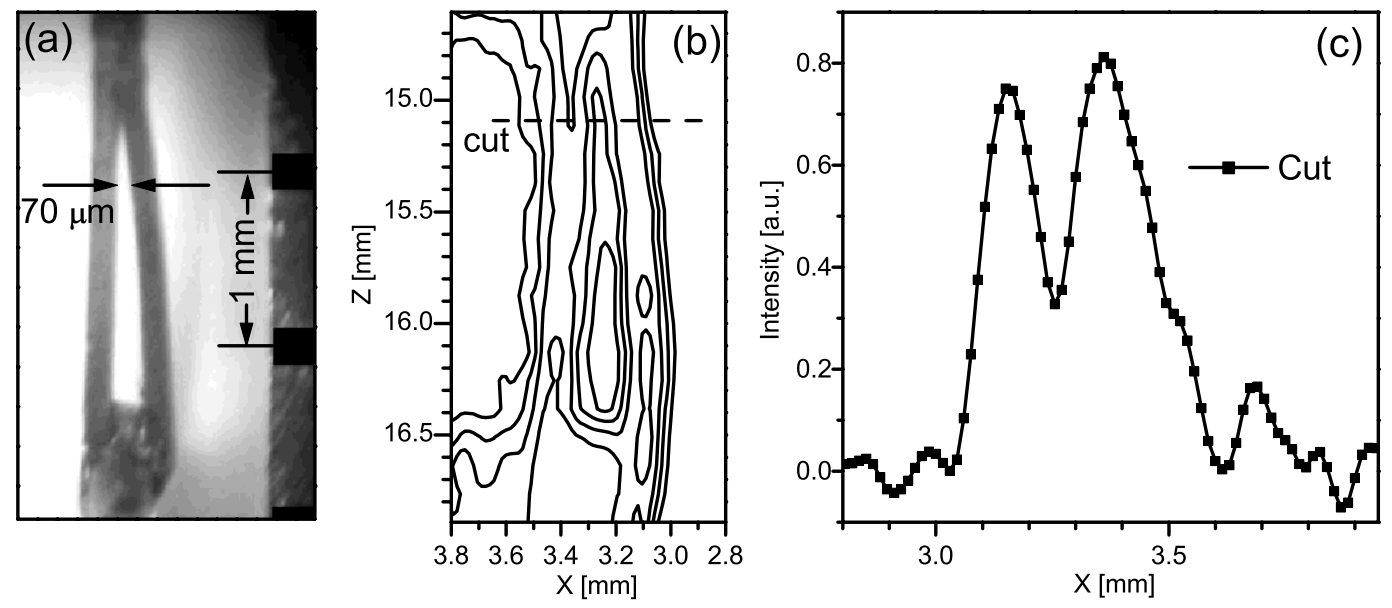

Figure 3. Measurement of the axial resolution. (a) Image and (b) measurement of an object showing the axial resolution. (c) 1D axial profile of intensity from the data in (b). 

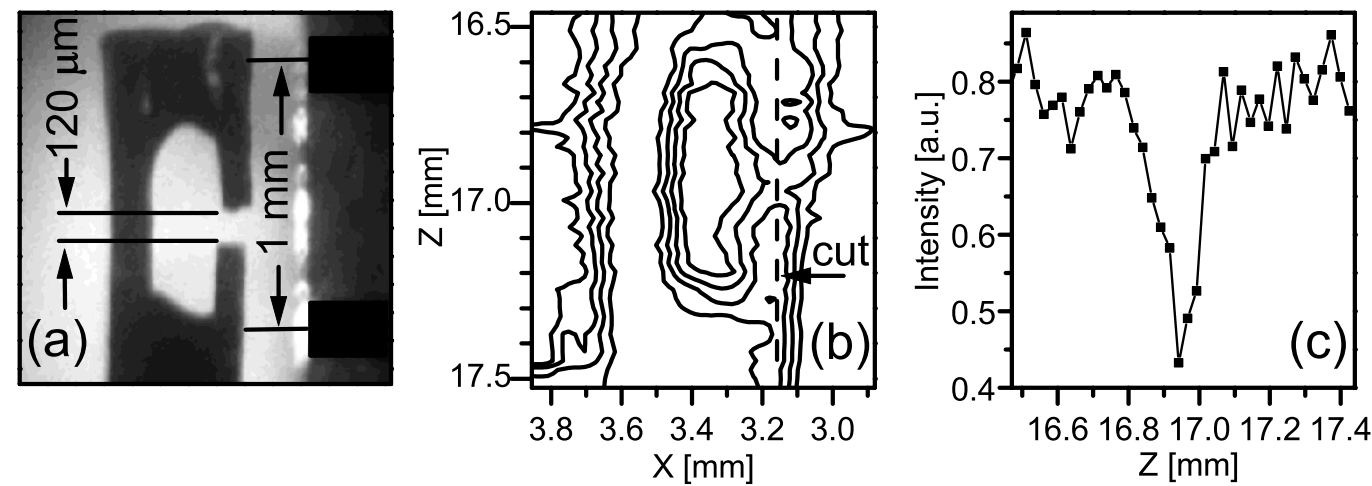

Figure 4. Measurement of the lateral resolution. (a) Image and (b) measurement of an object showing the lateral resolution. (c) 1D lateral profile of intensity from the data in (b).

To investigate the axial resolution, we imaged a light absorbing object in a chicken breast tissue sample (Fig. 3). The sample was prepared with 3.2-mm radii of curvature in the cylindrical bump. The object shown in Fig. 3(a) was made of 100- $\mu \mathrm{m}$ thick black latex sheet and placed in the center of the curvature of the prepared sample, i.e., $3.2 \mathrm{~mm}$ below the surface of the sample. The wide side of the object was parallel to the ultrasound beam and perpendicular to the light beam. We took the difference between the profiles of the modulated intensity along the $\mathrm{X}$ axis and the typical profile without object present and, subsequently, divided the difference by the latter profile point-by-point to obtain the relative values, which are shown as a contour-plot image with five equally spaced levels between 0 and 1 [Fig. 3(b)]. Figure 3(c) presents the $1 \mathrm{D}$ axial intensity profile along the $\mathrm{X}$ axis taken from the image in Fig. 3(b) at position $\mathrm{Z}=15.11 \mathrm{~mm}$, with an arbitrary origin. At position $\mathrm{Z}=15.11$ $\mathrm{mm}$, the gap had an actual width of only $70 \mu \mathrm{m}$ along the $\mathrm{X}$ axis and was resolved with a $55 \%$ contrast.

Similarly, to investigate the lateral resolution, the sample was prepared with 3-mm radii of curvature in the cylindrical bump. The object shown in Fig. 4(a) was also made of $100-\mu \mathrm{m}$ thick black latex sheet and placed in the center of the curvature of the prepared sample, $3 \mathrm{~mm}$ below the surface of the sample, with wide side parallel to the ultrasound beam and perpendicular to the light beam. The relative profile of the measured modulated optical intensity is shown as a contour-plot image with five equally spaced levels between 0 and 1 [Fig. 4(b)]. Figure 4(c) presents the 1D lateral intensity profile along the $\mathrm{Z}$ axis taken from the image in Fig. 4(b) at $\mathrm{X}=3.17$ $\mathrm{mm}$. The gap had an actual width of $120 \mu \mathrm{m}$ along the $\mathrm{Z}$ axis and was resolved with a $50 \%$ contrast. If we use the minimal sizes of the resolvable gaps at a $50 \%$ contrast as the resolutions, the estimated axial and lateral resolutions are $70 \mu \mathrm{m}$ and $120 \mu \mathrm{m}$, respectively. However, the ultimate resolvable gap sizes at minimal contrast should be much smaller.

\section{CONCLUSION}

In summary, this study demonstrated the feasibility of high-resolution ultrasound-modulated optical tomography in biological tissue with an imaging depth of several millimeters. A CFPI was shown to be able to efficiently isolate ultrasonically modulated light from the background in real time. The resolution can be further improved by using higher ultrasound frequencies. This technology can be easily integrated with conventional ultrasound imaging to provide complementary information.

We thank J. Li and K. Maslov for their fruitful scientific discussions. This research was supported by the National Institute of Health. L. Wang's e-mail address is lwang@tamu.edu.

\section{REFERENCES}

1. F. A. Marks, H. W. Tomlinson, and G. W. Brooksby, "A comprehensive approach to brest cancer detection using light: photon localization by ultrasound modulation and tissue characterization by spectral discrimination," in Proc. Soc. Photo-Opt. Instrum. Eng., 1888, pp. 500-510, 1993. 
2. L.-H. V. Wang, S. L. Jacques, and X. Zhao, "Continuous-wave ultrasonic modulation of scattered laser light to image objects in turbid media," Opt. Lett. 20, pp. 629-631, 1995.

3. W. Leutz and G. Maret, "Ultrasonic modulation of multiply scattered-light," Physica B 204, pp. 14-19, 1995.

4. L.-H. V. Wang, "Mechanisms of ultrasonic modulation of multiply scattered coherent light: an analytic model," Phys. Rev. Lett. 87, pp. 043903-(1-4), 2001.

5. S. Sakadzic and L.-H. V. Wang, "Ultrasonic modulation of multiply scattered coherent light: an analytical model for anisotropically scattering media," Phys. Rev. E 66, pp. 026603-(19), 2002.

6. M. Kempe, M. Larionov, D. Zaslavsky, and A. Z. Genack, "Acousto-optic tomography with multiply scattered light," J. Opt. Soc. Am. A 14, pp. 1151-1158, 1997.

7. L.-H. V. Wang and G. Ku, "Frequency-swept ultrasound-modulated optical tomography of scattering media," Opt. Lett. 23, pp. 975-977, 1998.

8. S. Leveque, A. C. Boccara, M. Lebec, and H. Saint-Jalmes, "Ultrasonic tagging of photon paths in scattering media: parallel speckle modulation processing," Opt. Lett. 24, pp. 181-183, 1999.

9. G. Yao, S.-L. Jiao, and L.-H. V. Wang, "Frequency-swept ultrasound-modulated optical tomography in biological tissue by use of parallel detection," Opt. Lett. 25, pp. 734-736, 2000.

10. A. Lev, Z. Kotler, and B. G. Sfez, "Ultrasound tagged light imaging in turbid media in a reflectance geometry," Opt. Lett. 25, pp. 378-380, 2000.

11. M. Hisaka, T. Sugiura, and S. Kawata, "Optical cross-sectional imaging with pulse ultrasound wave assistance," J. Opt. Soc. Am. A-Opt. Image Sci. Vis. 18, pp. 1531-1534, 2001.

12. J. Li, G. Ku, and L.-H. V. Wang, "Ultrasound-modulated optical tomography of biological tissue by use of contrast of laser speckles," Appl. Optics 41, pp. 6030-6035, 2002.

13. A. Lev and B. G. Sfez, "Pulsed ultrasound-modulated light tomography," Opt. Lett. 28, pp. 1549-1551, 2003.

14. M. Gross, P. Goy, and M. Al-Koussa, "Shot-noise detection of ultrasound-tagged photons in ultrasoundmodulated optical imaging," Opt. Lett. 28, pp. 2482-2484, 2003.

15. J. Li and L.-H. V. Wang, "Ultrasound-modulated optical computed tomography of biological tissues," Appl. Phys. Lett 84, pp. 1597-1599, 2004.

16. J.-P. Monchalin, "Optical detection of ultrasound at a distance using a confocal fabry-perot interferometer," Appl. Phys. Lett 47, pp. 14-16, 1985.

17. "Mammalian in vivo ultrasonic biological effects," http://www.aium.org/, 1992. 